\title{
Acesso a serviços de saúde por ribeirinhos de um município no interior do estado do Amazonas, Brasil
}

\section{Access to health services for riverside residents in a municipality in Amazonas State, Brazil}

Ananias Facundes Guimarães (iD), Victor Linec Maciel Barbosaㄹ (iD, Mariana Paula da Silva' (D), Jéssica Karoline Alves Portugal' (iD), Marcelo Henrique da Silva Reis' (iD), Abel Santiago Muri Gama' (iD)

' Universidade Federal do Amazonas, Instituto de Saúde e Biotecnologia, Coari, Amazonas, Brasil

\begin{abstract}
RESUMO
OBJETIVO: Descrever as características do acesso a serviços de saúde por ribeirinhos do município de Coari, estado do Amazonas, Brasil. MÉTODOS: Estudo descritivo, transversal, de base populacional, conduzido com ribeirinhos residentes na zona rural de Coari, no período de abril a julho de 2015. As entrevistas foram realizadas por meio de questionário previamente elaborado. Foram utilizadas variáveis socioeconômicas, demográficas e de acesso a serviços de saúde. A amostra probabilística por conglomerados foi composta por 492 sujeitos. RESULTADOS: Mais da metade (54,9\%) dos ribeirinhos utilizavam pequenas embarcações de madeira para acessar os serviços de saúde, navegando em média 60 km e tempo médio de viagem de 4,2 h. O serviço de saúde mais procurado pelos ribeirinhos foi o hospital (65,0\%), seguido da Unidade Básica de Saúde (26,6\%) e de farmácias (5,4\%). Cerca de um em cada quatro ribeirinhos (22,2\%) afirmaram tentar agendar uma consulta médica sem êxito, tendo como principais motivos a falta de fichas (vagas) para agendamento $(57,8 \%)$, a falta de profissionais para atendimento $(28,4 \%)$ e a demora no atendimento para a marcação de consulta (13,8\%). CONCLUSÃO: $\bigcirc$ acesso a serviços de saúde pelos ribeirinhos é limitado, sobretudo por barreiras geográficas. É necessária a articulação entre estratégias que promovam o acesso aos serviços de saúde pelos ribeirinhos, viabilizando a continuidade da assistência a saúde a essa população.
\end{abstract}

Palavras-chave: População Rural; Populações Vulneráveis; Acesso Universal aos Serviços de Saúde.

\begin{abstract}
OBJECTIVE: To describe the characteristics of access to health services for riverside dwellers in the Municipality of Coari, Amazonas State, Brazil. METHODS: Descriptive, cross-sectional, population-based study was conducted with riverside dwellers in the rural area of Coari, from April to July 2015. The interviews were conducted using a previously prepared questionnaire. Socioeconomic, demographic and access to health services variables were used. The probabilistic sample by clusters was composed of 492 subjects. RESULTS: More than half (54.9\%) of the riverside inhabitants used small wooden boats to access health services, sailing the average of $60 \mathrm{~km}$ and of $4.2 \mathrm{~h}$ travel time. The health service most sought by those populations was hospital (65.0\%), followed by Basic Health Unit (26.6\%), and pharmacies (5.4\%). About one in four riverside dwellers (22.2\%) stated that they tried unsuccessfully to book a medical appointment, with the main reasons being there were no appointments available (57.8\%), the lack of professionals to assist them (28.4\%), and the delay to get appointments (13.8\%). CONCLUSION: The access to health services for riverside dwellers is limited, especially by geographical barriers. It is necessary to articulate strategies that promote access to health services, enabling the continuity of health care to this population.
\end{abstract}

Keywords: Rural Population; Vulnerable Populations; Universal Access to Health Services.

\section{Correspondência / Correspondence:}

Abel Santiago Muri Gama

Estrada Coari Mamiá, 305. Bairro: Espírito Santo. CEP: 69460-000 - Coari, Amazonas, Brasil - Tel.: +55 (97) 98121-9295

E-mail: abelsmg@hotmail.com 


\section{INTRODUÇÃO}

A Amazônia brasileira possui uma superfície aproximada de $5.217 .423 \mathrm{~km}^{2}$, correspondente a cerca de $61 \%$ do território brasileiro, dentre os quais cerca de um terço $\left(1,6\right.$ milhões de $\left.\mathrm{km}^{2}\right)$ pertence ao maior estado do Brasil, o Amazonas. Inversamente proporcional à extensa área territorial, o Estado apresenta a segunda menor densidade demográfica do país $\left(2,2 \text { habitantes por } \mathrm{km}^{2}\right)^{1}$.

A dispersão demográfica e o vasto território, banhado por extensas bacias hidrográficas e coberto pela maior floresta tropical do planeta, impõem severas desigualdades de acesso a saúde e outras distorções sociais em relação às demais regiões brasileiras ${ }^{2}$.

As populações ribeirinhas do Amazonas são descendentes de povos indígenas mesclados com nordestinos e outros migrantes e vivem às margens dos rios e lagos ${ }^{3}$. Esses habitantes sobrevivem de pesca, caça, agricultura familiar e subsídios oriundos de programas sociais do governo federal. Suas comunidades carecem de recursos básicos, como saneamento básico e eletricidade, e dependem das áreas urbanas para aquisição de bens de consumo e assistência a saúde. $\bigcirc$ deslocamento até as áreas urbanas é por via fluvial, realizado com pequenas embarcações, em viagens que podem durar de alguns minutos a dias de navegação². Em conjunto com as condições adversas da região, os ribeirinhos estão expostos a doenças infecciosas (malária, parasitoses, doença de Chagas) ${ }^{4}$ associadas a morbidades típicas dos grandes centros urbanos, como hipertensão e diabetes ${ }^{5}$.

Embora a Constituição Federal de 1988 garanta o direito de acesso universal e igualitário a saúde a todos os brasileiros ${ }^{6}$, há evidências de que o acesso venha sendo um desafio para os usuários do Sistema Único de Saúde (SUS).

Um estudo realizado, em âmbito nacional e com dados da Pesquisa Nacional por Amostra de Domicílios (PNAD), excluindo a população rural dos estados da Região Norte, apontou que o principal motivo da não obtenção de atendimento a saúde foi não ter conseguido vaga ou senha e não haver médicos para esse atendimento ${ }^{7}$.

$\mathrm{Na}$ região metropolitana de Belo Horizonte, profissionais de saúde apontaram alguns fatores que dificultam o acesso a saúde pela população: a distância da residência do usuário à Unidade Básica de Saúde (UBS), a deficiência do transporte público no município e a impossibilidade do pagamento do transporte devido às dificuldades econômicas dos usuários ${ }^{8}$.

Na Região Norte, uma investigação realizada com pescadores ribeirinhos do Rio Machado do Ji-Paraná, no estado de Rondônia, apontou que, ao buscarem um serviço de saúde, os maiores problemas enfrentados foram a falta de médicos especialistas, a demora para marcar consultas e exames específicos e a falta de medicamentos nas UBS?.

As limitações de acesso aos serviços de saúde compõem um contexto multifatorial, complexo e subjetivo $^{10}$, que podem implicar em diferentes aspectos no modo de vida dessas populações. Nesse sentido, o objetivo deste estudo foi descrever as características do acesso a serviços de saúde por ribeirinhos do município de Coari, no Amazonas.

\section{MATERIAIS E MÉTODOS}

Este estudo faz parte da pesquisa "Saúde, Medicamentos e Automedicação em Ribeirinhos do Amazonas" (SAMARA).

Trata-se de um estudo transversal, de base populacional, conduzido com ribeirinhos residentes na zona rural de Coari, realizado no período de abril a julho de 2015.

Coari está localizado na região central do estado do Amazonas, na calha média do Rio Solimões, distante a $363 \mathrm{~km}$ de Manaus. $\bigcirc$ acesso ao município é realizado por via fluvial (de 9 a $30 \mathrm{~h}$ de viagem, dependendo da embarcação) ou aérea (1 h até Manaus). A população estimada, no ano de $2017^{1}$, era de 84.762 habitantes. A área territorial de Coari é de 57.970 km² e a densidade demográfica de 1,3 hab. $/ \mathrm{km}^{2}$.

Os principais serviços de saúde de Coari são um hospital de média complexidade (105 leitos), 12 UBS, um Instituto Tropical de Medicina, uma UBS Fluvial, um barco hospital para cobrir todas as comunidades ribeirinhas, um Laboratório Central de Análises Clínicas, um Serviço de Emergência, um Núcleo de Vigilância Sanitária, uma Policlínica e um Centro de Atenção Psicossocial ${ }^{11}$.

Os habitantes da zona rural estão distribuídos em comunidades dispersas ao longo das margens do rio Solimões, lagos e igarapés, em áreas de terra firme e várzeas.

A amostra calculada foi de 470 sujeitos, em função do número de comunidades ribeirinhas cobertas pela Secretaria Municipal de Saúde de Coari $(N=135)$, de modo a garantir a representatividade da área territorial da pesquisa e do número de habitantes adultos $(N=10.333)$. Adotou-se a técnica de amostragem probabilística por conglomerados, cujos detalhes do cálculo amostral podem ser visualizados em publicação prévia $^{2}$. Foram incluídos indivíduos com 18 anos de idade ou mais que se encontravam na residência no momento da aplicação do questionário.

Nesta investigação, foram coletadas informações socioeconômicas, demográficas e de acesso aos serviços de saúde. Os questionários foram aplicados por entrevistadores previamente treinados do Instituto de Saúde e Biotecnologia da Universidade Federal do Amazonas. Os ribeirinhos foram abordados em suas residências e convidados a participar do estudo.

As variáveis analisadas foram as de natureza demográfica (sexo, idade, estado conjugal, moradores por domicílio), socioeconômica (escolaridade, renda familiar mensal, atividade laboral) e de acesso aos serviços de saúde (distância e tempo para acesso aos serviços de saúde, procura pelos serviços de saúde nos últimos 30 dias, transporte comumente utilizado para 
acesso, tipo de profissional e de serviço que procura em caso de doença, tentativa de agendar consultas e motivo pelo qual não conseguiu o agendamento.

Os dados foram digitados a partir de uma máscara de inserção de dados no software SPSS (Statistical Package for Social Sciences) v22.0 para Windows. As variáveis qualitativas foram descritas por meio do cálculo de frequências absolutas e relativas; e, para as variáveis quantitativas, foram calculadas as medidas de tendência central.

Os indivíduos que consentiram a participação neste estudo assinaram o Termo de Consentimento Livre e Esclarecido. Aos que não sabiam assinar, foram disponibilizados tinteiros para coletar as digitais dos polegares.

estudo foi aprovado pelo Comitê de Ética em Pesquisa da Escola de Enfermagem da Universidade de São Paulo, sob parecer $n^{\circ}$ 33560914.0.0000.5392, em 5 de agosto de 2014.

Tabela 1 - Distribuição dos sujeitos da pesquisa segundo variáveis socioeconômicas e demográficas, Coari, estado do Amazonas, Brasil, 2015

\begin{tabular}{|c|c|c|}
\hline \multirow{2}{*}{ Variáveis } & \multicolumn{2}{|c|}{ Ribeirinhos } \\
\hline & $N=492$ & $\%$ \\
\hline \multicolumn{3}{|l|}{ Sexo } \\
\hline Masculino & 231 & 47,0 \\
\hline Feminino & 261 & 53,0 \\
\hline \multicolumn{3}{|l|}{ Idade (anos) } \\
\hline 18 a 39 & 287 & 58,3 \\
\hline 40 a 59 & 149 & 30,3 \\
\hline$\geq 60$ & 56 & 11,4 \\
\hline \multicolumn{3}{|l|}{ Analfabeto } \\
\hline Sim & 109 & 22,2 \\
\hline Não & 383 & 77,8 \\
\hline \multicolumn{3}{|c|}{ Escolaridade (anos de estudo) } \\
\hline Não estudou & 48 & 9,7 \\
\hline 1 a 4 & 146 & 29,7 \\
\hline 5 a 9 & 143 & 29,1 \\
\hline$\geq 10$ & 155 & 31,5 \\
\hline \multicolumn{3}{|l|}{ Estado conjugal } \\
\hline Vive só & 94 & 19,1 \\
\hline Vive acompanhado & 398 & 80,9 \\
\hline \multicolumn{3}{|l|}{ Atividade laboral } \\
\hline Não exerce atividade & 103 & 20,9 \\
\hline Agricultura e pesca & 311 & 63,2 \\
\hline Funcionário público* & 59 & 12,0 \\
\hline Outras & 19 & 3,9 \\
\hline \multicolumn{3}{|c|}{ Renda familiar mensal $(S M)^{\dagger}$} \\
\hline$<1$ & 205 & 41,7 \\
\hline 1 a 2 & 195 & 39,6 \\
\hline$>2$ & 92 & 18,7 \\
\hline \multicolumn{3}{|l|}{$\begin{array}{l}\text { Número de moradores por } \\
\text { domicílio }\end{array}$} \\
\hline 1 a 5 & 246 & 50,0 \\
\hline$\geq 6$ & 246 & 50,0 \\
\hline
\end{tabular}

* Funcionário público: professor, agente comunitário de saúde e merendeira; † SM: salário mínimo referente a abril de 2015 (R\$788,00).

\section{RESULTADOS}

Foram entrevistados 492 ribeirinhos, dentre os quais $53,0 \%$ pertenciam ao sexo feminino, $88,6 \%$ eram adultos, 58,8\% possuíam baixa escolaridade e 63,2\% apresentavam a pesca e a agricultura como atividade econômica. Quase a metade dos ribeirinhos apresentou renda familiar inferior a um salário mínimo $(41,7 \%)$ (Tabela 1).

Dentre os entrevistados, mais da metade $(54,9 \%)$ dos ribeirinhos utilizava pequenas embarcações de madeira, movidas a motor (rabeta), para acessar os serviços de saúde, navegando em média 60,4km, com tempo médio de viagem de 4,2 h (Tabela 2).

Quanto ao tipo de profissional mais procurado em caso de problemas de saúde, 42,7\% afirmaram a procura por médicos, seguidos dos agentes comunitários de saúde $(36,2 \%)$ e farmacêuticos $(5,3 \%)$. Em relação aos serviços de saúde mais procurados, $65,0 \%$ afirmaram procurar atendimento diretamente no hospital da cidade, seguido pela UBS $(26,6 \%)$ e farmácias (5,5\%). Dentre os entrevistados, 22,2\% dos ribeirinhos afirmaram que já tentaram marcar consultas sem sucesso e os motivos mais citados foram a falta de fichas (vagas) para agendamento $(57,8 \%)$, falta de profissionais para atendimento $(28,4 \%)$ e demora no atendimento para a marcação da consulta (13,8\%) (Tabela 3).

Tabela 2 - Distribuição dos sujeitos da pesquisa segundo variáveis relativas à maneira de acesso aos serviços de saúde, município de Coari, estado do Amazonas, Brasil, 2015

$\begin{array}{lll}\text { Variáveis } & \mathrm{N}=492 \quad \%\end{array}$

Transporte utilizado para acessar

os serviços de saúde

$\begin{array}{lrr}\text { Rabeta* }^{*} & 270 & 54,9 \\ \text { Recreio }^{\dagger} & 169 & 34,4 \\ \text { Motocicleta } & 17 & 3,5 \\ \text { Canoa a remo } & 15 & 3,0 \\ \text { Lancha comunitária } & 11 & 2,2 \\ \text { Outros }^{\ddagger} & 10 & 2,0\end{array}$

Distância da comunidade ao serviço de saúde $(\mathrm{km})$

\begin{tabular}{lll}
$<50$ & 253 & 51,4 \\
50 a 100 & 139 & 28,3 \\
$>100$ & 100 & 20,3 \\
Média & \multicolumn{2}{c}{60,4}
\end{tabular}

Tempo de deslocamento da comunidade ao serviço de saúde (hora)

$\begin{array}{lrl}<1 & 54 & 11,0 \\ 1 \text { a } 4 & 251 & 51,0 \\ >4 & 187 & 38,0\end{array}$

Média 4,2

* Pequena embarcação de madeira movida a motor; ${ }^{\dagger}$ Embarcação de grande porte; ${ }^{\ddagger}$ Outros: caminhão, a pé. 
Tabela 3 - Distribuição dos sujeitos da pesquisa segundo variáveis relativas ao modo de acesso aos serviços de saúde, Coari, estado do Amazonas, Brasil, 2015

\begin{tabular}{|c|c|c|}
\hline \multirow{2}{*}{ Variáveis } & \multicolumn{2}{|c|}{ Ribeirinhos } \\
\hline & $N=492$ & $\%$ \\
\hline \multicolumn{3}{|l|}{ Quem procura em caso de doença } \\
\hline Médico & 210 & 42,7 \\
\hline Agente comunitário de saúde & 178 & 36,2 \\
\hline Farmacêutico & 26 & 5,3 \\
\hline Enfermeiro & 23 & 4,7 \\
\hline Amigo, vizinho ou conhecido & 23 & 4,7 \\
\hline Não procura & 16 & 3,2 \\
\hline Outro* & 16 & 3,2 \\
\hline \multicolumn{3}{|l|}{ Estabelecimento de saúde que procura em caso de doença } \\
\hline Hospital Regional de Coari & 320 & 65,0 \\
\hline UBS ribeirinha & 131 & 26,6 \\
\hline Farmácias & 27 & 5,5 \\
\hline Outras $^{\dagger}$ & 14 & 2,9 \\
\hline \multicolumn{3}{|l|}{ Tentativa de agendar consulta sem êxito } \\
\hline Sim & 109 & 22,2 \\
\hline Não & 367 & 74,6 \\
\hline Nunca agendou & 16 & 3,2 \\
\hline \multicolumn{3}{|l|}{ Motivo pelo qual não conseguiu $(\mathrm{N}=109)$} \\
\hline Falta de fichas (vagas) para agendamento & 63 & 57,8 \\
\hline Falta de profissionais para atendimento & 31 & 28,4 \\
\hline Demora no atendimento para a marcação da consulta & 15 & 13,8 \\
\hline
\end{tabular}

* Rezador/pegador: curandeiro local; ${ }^{\dagger}$ Instituto de Medicina Tropical de Coari; clínica particular.

\section{DISCUSSÃO}

A população ribeirinha de Coari apresentou baixa escolaridade e nível econômico. As principais atividades exercidas foram a agricultura e a pesca; tendo, como principal meio de transporte, embarcações rústicas movidas a motor (rabeta) que percorrem longas distâncias até o acesso à zona urbana.

A baixa escolaridade dos ribeirinhos pode implicar diretamente no modo pelo qual sua saúde é gerida. Além disso, retrata a necessidade de ações específicas que considerem a realidade local e o contexto educacional.

Historicamente, populações rurais em todo mundo são destituídas de condições favoráveis à melhoria da qualidade de vida, o que inclui o acesso a educação, saúde, água potável e saneamento. O Relatório de Metas de Desenvolvimento do Milênio de 2015 apontou que, nas populações rurais de países em desenvolvimento, as crianças têm quatro vezes mais chances de não frequentarem escolas, e as taxas de mortalidade são, quase duas vezes, mais elevadas em relação às crianças de famílias com condições econômicas favoráveis. Além disso, nessas áreas, apenas $56,0 \%$ dos nascimentos são acompanhados por profissionais de saúde especializados, enquanto o percentual é de $87,0 \%$ nas áreas urbanas. Outro aspecto preponderante é o acesso a fontes de água potável (quatro vezes menor) e acesso a saneamento (três vezes menor) na zona rural em comparação à zona urbana ${ }^{12}$.
As limitações educacionais também impedem o desenvolvimento econômico e, por consequência, - acesso a bens de consumo (medicamentos, alimentação, planos de saúde), impactando negativamente na expectativa de vida. Ademais, a saúde preventiva é dificultada pelas limitações de entendimento do processo saúde-doença e do modo de prevenção de morbidades e promoção da saúde ${ }^{13}$.

No contexto sanitário, limitações educacionais contribuem para elevadas taxas de morbimortalidade por doenças evitáveis de cunho parasitário. Somente entre os anos de 2001 a 2009, o Brasil registrou 13.449 óbitos por doenças relacionadas ao saneamento básico inadequado, correspondendo a $1,31 \%$ do total de óbitos no período e cerca de 2,1 bilhões de reais em gastos do SUS com consultas médicas e internações hospitalares ${ }^{14}$.

A Amazônia brasileira apresenta um rol de doenças infecciosas típicas da região, e outras crônicas advindas das mudanças de hábitos alimentares nos últimos anos ${ }^{5}$, propiciando um ambiente preocupante no que tange ao controle e à prevenção dessas doenças. Muitas comunidades não possuem energia elétrica, limitando o acesso a informações veiculadas por internet, rádio e televisão. Além disso, não há saneamento básico e o acesso à água tratada é realizado apenas pelo uso de hipoclorito, nem sempre disponível ou com orientação inadequada quanto ao seu modo de uso, podendo levar à falsa impressão de prevenção². 
No que concerne ao baixo nível econômico dos ribeirinhos, é importante discutir o impacto de qualquer problema de saúde e o ônus econômico sobre o indivíduo e sua família. Há de se considerar a distância para acessar os serviços de saúde (média de $60 \mathrm{~km}$ ), a duração da viagem (média de $4 \mathrm{~h}$ ), os custos com deslocamento (as pequenas embarcações de madeira requerem combustível) e, nos casos de internações, os custos com hospedagem e alimentação dos acompanhantes, pois os ribeirinhos geralmente não possuem residência na zona urbana; além do impacto provocado pela necessidade de interromper as atividades de agricultura, pesca, entre outras, por vezes, as únicas fontes de renda nas comunidades. A soma dessas questões pode afetar a renda iá limitada dos ribeirinhos e no desfecho do problema de saúde, forçando os mesmos a recorrerem aos recursos disponíveis na própria comunidade ${ }^{15}$, podendo agravar o problema de base, na tentativa de solucionar ou protelar a procura pelo serviço de saúde na expectativa de melhoria do quadro mórbido.

Outros estudos realizados em populações ribeirinhas da Amazônia evidenciaram que as questões geográficas interferem diretamente no acesso aos serviços de saúde $2,15,16$. Em outros países, como na Austrália, resguardadas as diferenças sociopolíticas, geográficas e culturais, as populações rurais também sofrem consequências negativas impostas pelas mesmas questões ${ }^{17}$, evidenciando que características geográficas podem limitar o alcance aos serviços de saúde por essas populações.

Algumas estratégias foram direcionadas pelo governo brasileiro, a fim de reduzir as desigualdades de acesso à saúde enfrentadas por populações vulneráveis, dentre as quais incluem-se as populações ribeirinhas da Amazônia. O Programa Mais Médicos foi criado, em 2013 (Medida Provisória n 621/2013), com o objetivo de suprir as necessidades de médicos nas regiões vulneráveis do Brasil ${ }^{18}$. Nesse mesmo ano, a Região Norte brasileira foi a que apresentou a menor relação de médicos para cada 1.000 habitantes (1,0 médico/1.000 habitantes), realidade agravada no estado do Amazonas, sobretudo no interior (0,2 médicos/1.000 habitantes), correspondendo a uma relação 10 vezes menor quando comparada à capital Manaus (2,0 médicos/1.000 habitantes) ${ }^{16}$. Entre 2013 e 2017, o Programa Mais Médicos contou com 18.240 médicos (62,6\% eram médicos cubanos), distribuídos em $72,8 \%$ dos municípios brasileiros ${ }^{18}$. Esse programa, notoriamente, trouxe maior cobertura de médicos em áreas de difícil acesso e maior vulnerabilidade; no entanto, isoladamente, é incapaz de preencher as lacunas na assistência de saúde a essas populações ${ }^{19,20}$.

Outra estratégia que possibilitou avanços no acesso a saúde pelas populações ribeirinhas foi a implementação da Política Nacional de Atenção Básica (PNAB) (Portaria MS/GM n 2.488/2011 e Portaria MS/GM n 2.490/2011). De acordo com a PNAB, os municípios da Amazônia Legal e Mato Grosso do Sul podem optar entre dois arranjos organizacionais para Equipes de Saúde da Família: as Equipes de Saúde da Família Ribeirinhas (ESFR) e as Equipes de Saúde da
Família Fluviais (ESFF), as quais deverão ser compostas por, no mínimo, um médico, um enfermeiro, um técnico ou auxiliar de enfermagem e de seis a 12 agentes comunitários de saúde (ACS) ${ }^{21}$.

Embora as estratégias levantadas possam ter avançado no contexto da redução de desigualdades de acesso a saúde entre as populações ribeirinhas do Amazonas, em 2016, foi aprovada a Emenda Constitucional no95 que prevê o congelamento dos gastos públicos pelas duas décadas seguintes, dentre os quais, os gastos com a saúde ${ }^{22}$. Nesse contexto, considerando a situação de vulnerabilidade já imposta às populações ribeirinhas na Amazônia, é possível que tal medida incorra em impactos dramáticos com a redução de investimentos e aumento das desigualdades socioeconômicas e de acesso a saúde.

Quando analisadas as procuras por tipo de serviço e de profissional de saúde, em caso de doença entre os ribeirinhos, os dados indicam a preferência pelo serviço hospitalar e atendimento médico. Isso pode estar relacionado à crença de que os profissionais médicos são os únicos capazes de resolver os problemas de saúde da população e ao fato de que os serviços ofertados pela atenção primária são limitados em sua resolutividade ${ }^{23}$. Por outro lado, as limitações de acesso a saúde e a possibilidade de processos mórbidos complexos, possivelmente agravados até a chegada ao serviço de saúde, podem levar à necessidade de atendimento especializado no hospital.

Deve-se considerar que, em função do funcionamento das UBS ser diurno e de haver a necessidade de agendamento de consultas, para um atendimento de urgência ou em outro horário, o único serviço de saúde disponível seria o hospital. Além disso, apesar de os ribeirinhos acessarem a zona urbana do município mensalmente ${ }^{2}$, o limitado tempo de permanência na mesma e o horário da procura pelo serviço possivelmente não coincidam com o funcionamento da UBS. Tais constatações advertem sobre a necessidade de atendimento com demanda livre, para contemplar as premissas do SUS de universalidade e integralidade no atendimento ${ }^{24}$.

Os ACS foram procurados com frequência pelos ribeirinhos para a resolução de morbidades. Como não há serviços de saúde nas comunidades ribeirinhas, os ACS são os únicos profissionais nessas localidades e atuam como intermediadores entre a população e a rede de serviços de saúde. Esses são moradores das comunidades e trabalham na Estratégia Saúde da Família cadastrando e acompanhando famílias, por meio da visita domiciliar, e ainda orientam e desenvolvem atividades de promoção da saúde, prevenção de doenças e agravos, junto à comunidade ${ }^{21,25}$

As farmácias representaram a terceira escolha em caso de problema de saúde, assim como os farmacêuticos. É possível que esses estabelecimentos substituam, em algumas situações, a procura pelos serviços de saúde, sobretudo em casos de morbidades de "fácil" resolutividade 26 , como problemas álgicos, muito prevalentes em populações ribeirinhas devido 
às atividades laborais intensas. Nesse ponto, há que se preocupar com o mascaramento de doenças, que pode dar origem a outras enfermidades mais graves. Além disso, a procura por farmácias pode incentivar o consumo de medicamentos, elevando seu estoque nas residências, propiciando o uso irracional e a automedicação nas comunidades ribeirinhas².

Cerca de um a cada quatro ribeirinhos não obtiveram êxito em agendar consultas quando procuraram os serviços de saúde na zona urbana do município, principalmente pela falta de fichas ou de profissionais para atendimento. Esses dados estão em consonância com os da PNAD, que apontou tais aspectos como os principais motivos de não conseguir atendimento a saúde pela população brasileira ${ }^{7}$. É válido ressaltar que a pesquisa supracitada excluiu a população rural dos estados da Região Norte, o que aponta a necessidade de estudos que compreendam o acesso aos serviços de saúde nessa região.

Dentre as limitações do presente estudo, considera-se que a captação de informações sobre o tempo e a distância de acesso aos serviços de saúde pelos ribeirinhos pode sofrer grandes variações em relação ao momento da investigação. Este estudo foi conduzido durante o período em que os lagos e o rio permitiam a navegação com facilidade (durante as cheias), diferentemente do que ocorre nos períodos de seca (entre agosto e novembro), o que resulta em isolamento de algumas comunidades e eleva substancialmente o tempo decorrido para acessar os serviços de saúde.

\section{CONCLUSÃO}

O acesso a serviços de saúde pelos ribeirinhos é realizado por meio de pequenas embarcações de madeira, que podem percorrer longas distâncias entre as comunidades até o serviço de saúde. Os ribeirinhos costumam procurar com maior frequência o hospital, sendo constatado que a dificuldade em se agendar o atendimento é um dos principais motivos que limitam o acesso às UBS.

É necessária a articulação entre estratégias que promovam o acesso aos serviços de saúde pelos ribeirinhos, viabilizando a continuidade da assistência à saúde dessas populações.

\section{CONTRIBUIÇÃO DOS AUTORES}

Guimarães AF, Barbosa VLM, Silva MP, Portugal JKA e Reis MHS contribuíram na aquisição dos dados, revisão e interpretação dos resultados. Gama ASM contribuiu na concepção do trabalho, aquisição, análise e interpretação de dados, redação e revisão crítica do conteúdo.

\section{APOIO FINANCEIRO}

Fundação de Amparo à Pesquisa do Estado do Amazonas.

\section{CONFLITOS DE INTERESSES}

Os autores declaram que não há conflitos de interesse relativos à realização da presente pesquisa.

\section{REFERÊNCIAS}

1 Instituto Brasileiro de Geografia e Estatística. Cadastro de municípios localizados na Amazônia Legal [Internet]. Rio de Janeiro: IBGE; c2016 [citado 2019 abr 5]. Disponível em: https://www. ibge.gov.br/geociencias/informacoes-ambientais/ vegetacao/15819-amazonia-legal. $h t m l$ t $t=0$-que-e.

2 Gama ASM, Fernandes TG, Parente RCP, Secoli SR. Inquérito de saúde em comunidades ribeirinhas do Amazonas, Brasil. Cad Saude Publica. 2018 fev;34(2):e00002817.

3 Fraxe TJP, Pereira HS, Witkoski AC, organizadores. Comunidades ribeirinhas amazônicas: modos de vida e uso dos recursos naturais. Manaus: EDUA; 2007.

4 Santos FS, Gama ASM, Fernandes AB, Reis Jr JDD, Guimarães J. Prevalência de enteroparasitismo em crianças de comunidades ribeirinhas do Município de Coari, no médio Solimões, Amazonas, Brasil. Rev Pan-Amaz Saude. 2010 dez; 1 (4):23-8.

5 Oliveira BFA, Mourão DS, Gomes N, Costa JMC, Souza AV, Bastos WR, et al. Prevalência de hipertensão arterial em comunidades ribeirinhas do Rio Madeira, Amazônia Ocidental Brasileira. Cad Saude Publica. 2013 ago;29(8):1617-30.
6 Brasil. Constituição (1988). Constituição da República Federativa do Brasil. Brasília, DF: Senado Federal; 1988.

7 Pinheiro RS, Viacava F, Travassos C, Brito AS. Gênero, morbidade, acesso e utilização de serviços de saúde no Brasil. Cienc Saude Coletiva. 2002;7(4):687-707.

8 Viegas APB, Carmo RF, Luz ZMP. Fatores que influenciam o acesso aos serviços de saúde na visão de profissionais e usuários de uma unidade básica de referência. Saude Soc. 2015 jan-mar;24(1):100-12.

9 Prosenewicz I, Lippi UG. Acesso aos serviços de saúde, condições de saúde e exposição aos fatores de risco: percepção dos pescadores ribeirinhos do Rio Machado de Ji-Paraná, RO. Saude Soc. 2012;21(1):219-31.

10 Assis MMA, Jesus WLA. Acesso aos serviços de saúde: abordagens, conceitos, políticas e modelo de análise. Cienc Saude Coletiva. 2012 nov; $17(11): 2865-75$. 
11 Ministério da Saúde (BR). Secretaria de Atenção à Saúde. Cadastro Nacional de Estabelecimentos de Saúde. Estabelecimento de saúde do município: Coari [Internet]. Brasília: Ministério da Saúde; 2016 [citado 2017 jun 7]. Disponível em: http://cnes2. datasus.gov.br/Lista_Es_Municipio.a

12 United Nations. The millennium development goals report: 2015 [Internet]. New York: United Nations; 2015. [cited 2017 Jun 7]. Available from: http:// mdgs.un.org/unsd/mdg/Resources/Static/Products/ Progress2015/English2015.pdf.

13 Molina-Salazar RE, Aguilar-Bustamantes F, Amozurrutia-Jiménez JA. Acceso con equidad en los servicios de salud en México un enfoque institucional. Horiz Sanitario. 2018;17(3):197-207.

14 Teixeira JC, Oliveira GS, Viali AM, Muniz SS. Estudo do impacto das deficiências de saneamento básico sobre a saúde pública no Brasil no período de 2001 a 2009. Eng Sanit Ambient. 2014 jan-mar; 19(1):87-96.

15 Sousa IS. As condições de vida e saneamento nas comunidades da área de influência do gasoduto Coari-Manaus em Manacapuru - AM. Hygeia. 2009 dez;5(9):88-98.

16 Silveira RP, Pinheiro R. Entendendo a necessidade de médicos no interior da Amazônia - Brasil. Rev Bras Educ Med. 2014 out-dez;38(4):451-9.

17 Mcgrail MR, Humphreys JS, Ward B. Accessing doctors at times of need-measuring the distance tolerance of rural residents for health-related travel. BMC Health Serv Res. 2015 May; 15:212.

18 Ministério da Saúde (BR). Secretaria de Gestão do Trabalho e da Educação na Saúde. Programa Mais Médicos. Brasília: Ministério da Saúde; 2017. 36 p.

19 Medina MG, Almeida PF, Lima JG, Moura D, Giovanella L. Programa Mais Médicos: mapeamento e análise da produção acadêmica no período 2013-2016 no Brasil. Saude Debate. 2018 set;42(esp):346-60.
20 Veras KB, Feitosa ANA, Quental OB, Seabra CAM, Gondim FSS. Impacto do programa mais médicos na atenção básica: uma análise de literatura. Rev Interdisciplin Saude. 2018 abr-jun;5(2):294-309.

21 Brasil. Ministério da Saúde. Portaria $n^{\circ} 2.488$, de 21 de outubro de 2011 . Aprova a Política Nacional de Atenção Básica, estabelecendo a revisão de diretrizes e normas para a organização da Atenção Básica, para a Estratégia Saúde da Família (ESF) e o Programa de Agentes Comunitários de Saúde (PACS). Brasília (DF), 2011 out 27; Seção 2:28. Disponível em: http://bvsms.saude.gov.

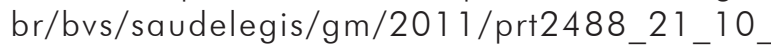
2011 .html.

22 Brasil. Emenda Constitucional $n^{\circ}$ 95, de 15 de dezembro de 2016. Altera o Ato das Disposições Constitucionais Transitórias, para instituir o Novo Regime Fiscal, e dá outras providências. Diário Oficial da União, Brasília (DF), 2016 dez 16; Seção 1:2. Disponível em: http://www.planalto. gov.br/ccivil_03/constituicao/emendas/emc/ emc95.htm.

23 Kassouf AL. Acesso aos serviços de saúde nas áreas urbana e rural do Brasil. Rev Econ Sociol Rural. 2005 jan-mar;43(1):29-44.

24 Brasil. Lei n 8.080, de 19 de setembro de 1990. Dispõem sobre as condições para a promoção, proteção e recuperação da saúde, a organização e o funcionamento dos serviços correspondentes e dá outras providências. Diário Oficial da União, Brasília (DF), 1990 set 20; Seção 1:18055.

25 Ministério da Saúde (BR). O trabalho do agente comunitário de saúde. Brasília: Ministério da Saúde; 2009. 80 p. (Série F. Comunicação e educação em saúde). [Link]

26 Matos JF, Pena DAC, Parreira MP, Santos TC, Coura-Vital W. Prevalência, perfil e fatores associados à automedicação em adolescentes e servidores de uma escola pública profissionalizante. Cad Saude Colet. 2018;26(1):76-83. 